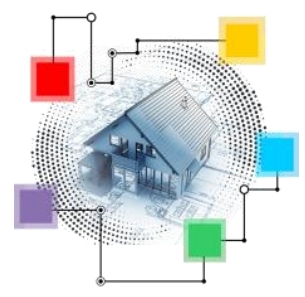

\author{
XIII SIMPÓSIO NACIONAL DE SISTEMAS PREDIAIS \\ DESEMPENHO E INOVAÇÃO \\ DE SISTEMAS PREDIAIS HIDRÁULICOS \\ SÃO PAULO - 04 DE OUTURO DE 2019
}

\title{
SISTEMA CONVENCIONAL X SISTEMA SIFÔNICO: UMA ANÁLISE TÉCNICO-ECONÔMICA NO USO EM SISTEMAS PREDIAIS DE ÁGUAS PLUVIAIS
}

\author{
CONVENTIONAL SYSTEM X SIFONIC SYSTEM: A TECHNICAL- \\ ECONOMIC ANALYSIS IN USE IN PREDIAL SYSTEMS OF PLUVIAL
}

\section{SASSO, Fabiane ${ }^{1}$; SCORTEGAGNA, Vinícius ${ }^{2}$; PAGNUSSAT, Rafaela Vicenzi ${ }^{3}$; FERNANDES, Vera ${ }^{4}$; FIORI, Simone ${ }^{5}$; LOPES, Anelise Sertoli ${ }^{6}$}

\author{
${ }^{1}$ Universidade de Passo Fundo, Av. Brasil Leste, 285 - São José, Passo Fundo, fabisasso@ hotmail.com \\ ${ }^{2}$ Universidade de Passo Fundo, viniciuss@upf.br \\ ${ }^{3}$ Universidade de Passo Fundo, rafaelavicenzi@upf.br \\ ${ }^{4}$ Universidade de Passo Fundo, cartana@upf.br \\ ${ }^{5}$ Universidade de Passo Fundo, sfiori@upf.br \\ ${ }^{6}$ Universidade de Passo Fundo, anelise.sertoli@upf.br
}

\begin{abstract}
RESUMO
Crescentemente o setor da construção civil demanda soluções eficientes e alinhadas com a inovação. No Brasil, os sistemas prediais de águas seguem os requisitos da NBR 10844/ (ABNT, 1989) onde os sistemas são concebidos como conduto livre. O sistema sifônico surge como alternativa, funcionando como conduto forçado. No presente estudo foram avaliadas duas edificações em uma cidade do norte do Rio Grande do Sul, sendo um galpão industrial com uma grande área de cobertura, e uma edificação hospitalar com um telhado menor. Os sistemas das duas edificações foram dimensionados com os índices pluviométricos de $125 \mathrm{~mm} / \mathrm{h}$ e $180 \mathrm{~mm} / \mathrm{h}$ e respectivos períodos de retorno de 5 e 25 anos. Analisaram-se ainda as características de ambos quanto ao processo construtivo e funcionamento. As informações adquiridas das características de cada um possibilitaram comparação técnicoeconômica dos custos, vantagens e desvantagens, considerando aplicabilidade em obras industriais e convencionais. Desenvolveu-se o levantamento de materiais e seus custos de implantação para as duas situações em cada uma das edificações. O sistema sifônico se destacou sendo um sistema mais completo, fácil montagem e eliminando grandes quantidades de condutores verticais das fachadas, porém este resultou em um custo de 6 à 8 vezes o custo do sistema convencional.
\end{abstract}

Palavras-chave: Águas Pluviais, Sistema Convencional, Sistema Sifônico, Comparação, TecnicoEconomicamente.

\begin{abstract}
Increasingly, the construction sector demands efficient solutions aligned with innovation. In Brazil, the building water systems follow the requirements of NBR 10844 / (ABNT, 1989) where the systems are designed as a free duct. The syphonic system appears as an alternative, acting as a forced conduit. In the present study, two buildings were evaluated in a city in northern Rio Grande do Sul, an industrial warehouse with a large coverage area, and a hospital building with a smaller roof. The systems of the two buildings were dimensioned with rainfall indices of $125 \mathrm{~mm} / \mathrm{h}$ and $180 \mathrm{~mm} / \mathrm{h}$ and respective return periods of 5 and 25 years. We also analyzed the characteristics of both regarding the construction process and operation. The acquired information of the characteristics of each one made possible a technical and economical comparison of the costs, advantages and disadvantages, considering applicability in industrial and conventional works. The survey of materials and their implementation costs for the two situations in each of the buildings was developed. The syphonic system stood out as a more complete system, easy assembly and eliminating large amounts of vertical conductors from the façades, but this resulted in a cost of 6 to 8 times the cost of the conventional system.
\end{abstract}

Keywords: Rainwater, Conventional System, Siphonic System, Comparation, Technical-Economically. 


\section{INTRODUÇÃO}

As edificações são compostas de sistemas que garantem o bom desempenho e conforto aos usuários. Destaca-se o sistema predial de águas pluviais, funcionando no transporte seguro dessas águas.

Os sistemas de coleta e disposição de águas pluviais são calculados em função da probabilidade de ocorrer precipitações extremas, em determinado período médio de vida útil do sistema analisado. Problemas na execução do sistema, como a não realização da declividade nas calhas, influência do traçado e, a não consideração de mudança de direção, gera problemas e possíveis transbordamentos no sistema.

Analisando pavilhões com grandes coberturas, sabe-se da ocorrência dos transbordamentos e vazamentos das calhas. Diante disto, avaliou-se o sistema sifônico, com funcionamento baseado em pressões negativas gerados pelos condutos quando submetidos ao efeito sifão. Ainda pouco difundido e sem normas brasileiras, será estudado com enfoque no dimensionamento e processo construtivo deste. Por isso, foi comparado técnico-economicamente o sistema sifônico e o sistema convencional preconizado pela NBR 10844/ (ABNT, 1989), considerando pontos de aplicação em obras industriais e convencionais, dimensionando calhas e condutores das coberturas, identificando vantagens e desvantagens, além de realizar as devidas comparações entre os sistemas, a fim de levantar quantitativos de custo. Para o sistema sifônico, os dados como dimensionamento e traçado para as edificações serão obtidos junto a empresa fabricante com cálculos através de softwares próprios.

\section{REVISÃO BIBLIOGRÁFICA}

\subsection{INSTALAÇÕES PREDIAIS DE ÁGUAS PLUVIAIS SEGUNDO DIRETRIZES BRASILEIRAS}

A NBR 10844/ (ABNT, 1989) exige critérios para instalações de drenagem de água pluvial, garantindo níveis adequados de funcionalidade. A área de contribuição deve ser calculada acrescentando resultantes inclinadas da cobertura e paredes que interceptam a água da chuva, drenadas pela cobertura.

Seguindo essa norma, deve-se fixar valores adequados para a duração de precipitação e período de retorno, a fim de determinar a intensidade pluviométrica para projetos. Para tal, tomou-se como base dados pluviométricos locais. A duração de precipitação é fixada em $t=5 \mathrm{~min}$. Já o período de retorno deve ser fixado, seguindo as características da área a ser drenada, obedecendo os critérios a seguir, para as situações utilizadas:

$\mathrm{T}=5$ anos, para coberturas e/ou terraços;

$\mathrm{T}=25$ anos, para coberturas e áreas onde o empoçamento ou extravasamento não possa ser tolerado.

\subsection{SISTEMA SIFÔNICO SEGUNDO DIRETRIZES DA EMPRESA EPAMS SAINT GOBAIN}

Uma característica do sistema sifônico é o escoamento em seção plena, impedindo a entrada de ar no interior dos tubos, formando pressão negativa e aumentado a velocidade de escoamento resultante da sucção. Isso permite que, para uma mesma vazão comparado ao sistema convencional, os diâmetros sejam menores e sem inclinação. A vazão dentro da tubulação aumenta conforme a altura da lâmina d'água dentro das calhas, consequentemente a velocidade de sucção. 
Para Valsir (2017), quando um sistema de drenagem sifônico atinge toda a seção do condutor horizontal, o "efeito sifão" resulta numa força motriz, dada pela altura d'água acumulada dentro da calha, sendo que a falta de ar no sistema permite que ele funcione cheio de água, usando a seção total do tubo, com fluxos mais rápidos comparado ao sistema convencional.

O sistema recolhe águas pluviais através de captadores que impedem a entrada de ar nas canalizações. Para calhas solicita-se a largura de $40 \mathrm{~cm}$ de base, para encaixe dos captadores. EPAMS, (2017).

Iniciada a precipitação, a água escoa pelo sistema sifônico, sem pressão, é recolhida pela tubulação, por conduto livre. Após aumento da precipitação, a água atinge a placa anti-vórtice instalada no captador, impedindo a entrada de ar e o sistema iniciar a operação através da sucção.

Para os captadores do sistema sifônico, seguiu-se diretrizes da empresa Epams Saint Gobain, a qual utilizou software próprio, mas se reservou o direito de não disponibilizar a memória de cálculo, fornecendo apenas os resultados.

\section{MATERIAIS E MÉTODOS}

O estudo analisou o escoamento em condutores de água pluvial de duas edificações com de intensidade pluviométrica de $125 \mathrm{~mm} / \mathrm{h}$ com período de retorno de 5 anos e $180 \mathrm{~mm} / \mathrm{h}$ com 25 anos. Pode-se observar no Quadro 01 a caracterização das coberturas das edificações.

QUADRO 01 - Dados de duas coberturas usadas no estudo.

\begin{tabular}{|c|c|c|}
\hline Porte & $\begin{array}{c}\text { Galpão Industrial } \\
\text { (Grande) }\end{array}$ & $\begin{array}{c}\text { Hospital } \\
\text { (Pequeno) }\end{array}$ \\
\hline Área Horizontal $\left(\mathbf{m}^{\mathbf{2}}\right)$ & 23.976 & 715 \\
\hline Inclinação da Cobertura (\%) & 5 & 35 \\
\hline Telha & $\begin{array}{c}\text { Zipada em telhas galvanizadas, } \\
\text { espessura 0,65mm }\end{array}$ & Telhas Shingle \\
\hline Altura do Condutor Vertical $(\mathbf{m})$ & 12 & 14,15 \\
\hline Material, espessura calhas & $\begin{array}{c}\text { Bobinas base galvanizada pré- } \\
\text { pintada 02 faces, 0,65 mm. }\end{array}$ & $\begin{array}{c}\text { Bobinas base galvanizada pré- } \\
\text { pintada 02 faces, } 0,65 \text { mm. }\end{array}$ \\
\hline
\end{tabular}

Fonte: Autores (2019).

Comparando os registros de precipitações pluviométricas anuais na estação meteorológica de Passo Fundo com a normal climatológica local, a maioria dos 6 anos analisados ficou cerca de $25 \%$ acima da média normal.

A empresa Epams Saint Gobain, dimensionou e levantou custos do sistema sifônico, com softwares próprios, possibilitando comparação com o sistema convencional, com dimensionamento, levantamento de materiais empregados e custos de acordo com a NBR 10844 (ABNT, 1989), enfatizando o custo x benefício. Os custos para a orçamentação dos modelos, não contempla mão-de-obra, a qual poderia variar consideravelmente.

Os custos dos condutores verticais em PVC reforçado para o sistema convencional, foram extraídos de tabelas do SINAPI (2017). Os custos do sistema sifônico foram fornecidos pela empresa parceira, sem considerar a mão-de-obra. 


\section{RESULTADOS E DISCUSSÕES}

\subsection{DIMENSIONAMENTO CONVENCIONAL - GALPÃO INDUSTRIAL}

As informações para dimensionamentos encontram-se na Figura 01.

FIGURA 01 - Cobertura do galpão industrial

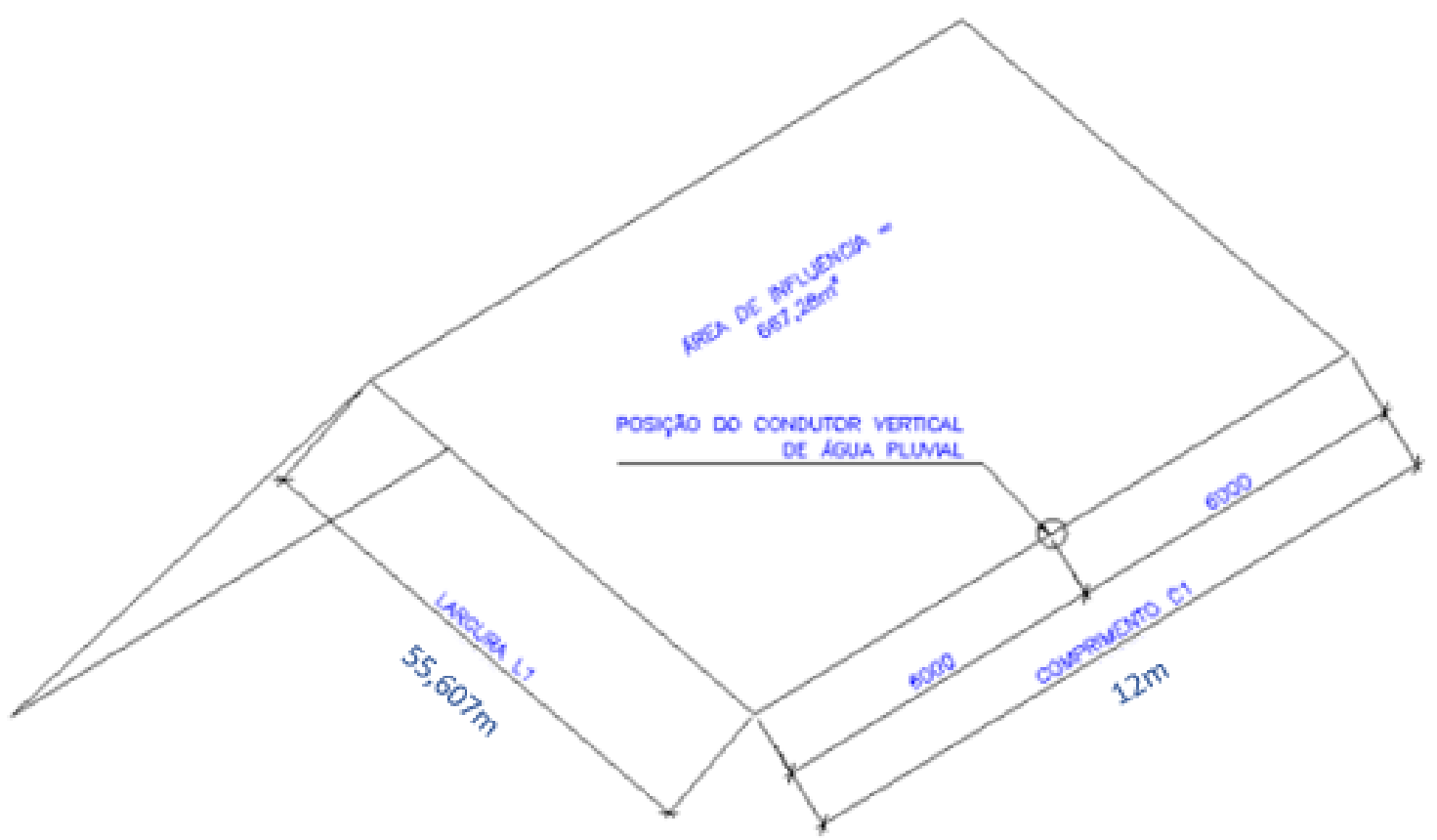

Fonte: Autores (2019).

\subsubsection{DIMENSIONAMENTO DOS CONDUTORES VERTICAIS E CALHAS}

Com intensidade pluviométrica de $125 \mathrm{~mm} / \mathrm{h}$, período de retorno de 5 anos e, considerando uma área de contribuição de $667,28 \mathrm{~m}^{2}$, obteve-se uma vazão de projeto de $1.390,17 \mathrm{~L} / \mathrm{min}$. Assim, por meio da NBR 10844 (ABNT, 1989) o diâmetro do condutor vertical corresponde a $150 \mathrm{~mm}$.

Para determinar a vazão das calhas, adotou-se uma base de $250 \mathrm{~mm}$ e altura de $100 \mathrm{~mm}$, suportando uma vazão de $1.403,90 \mathrm{~L} / \mathrm{min}$, é maior que a vazão de projeto.

Com intensidade de $180 \mathrm{~mm} / \mathrm{h}$, e período de retorno de 25 anos, a vazão de projeto obtida para a mesma cobertura é $2.001,84 \mathrm{~L} / \mathrm{min}$. Assim, o diâmetro do condutor vertical é $200 \mathrm{~mm}$. O dimensionamento da seção da calha corresponde a $300 \mathrm{~mm}$ de base e $110 \mathrm{~mm}$ de altura de lâmina d'água, suportando uma vazão de $2.025,03 \mathrm{~L} / \mathrm{min}$.

\subsection{DIMENSIONAMENTO CONVENCIONAL - COBERTURA HOSPITALAR}

As informações para dimensionamentos encontram-se na Figura 02. Considerando o posicionamento do condutor vertical na extremidade da calha, a área de maior contribuição é A02, com 106,17 m. 
FIGURA 02 - Cobertura da edificação hospitalar

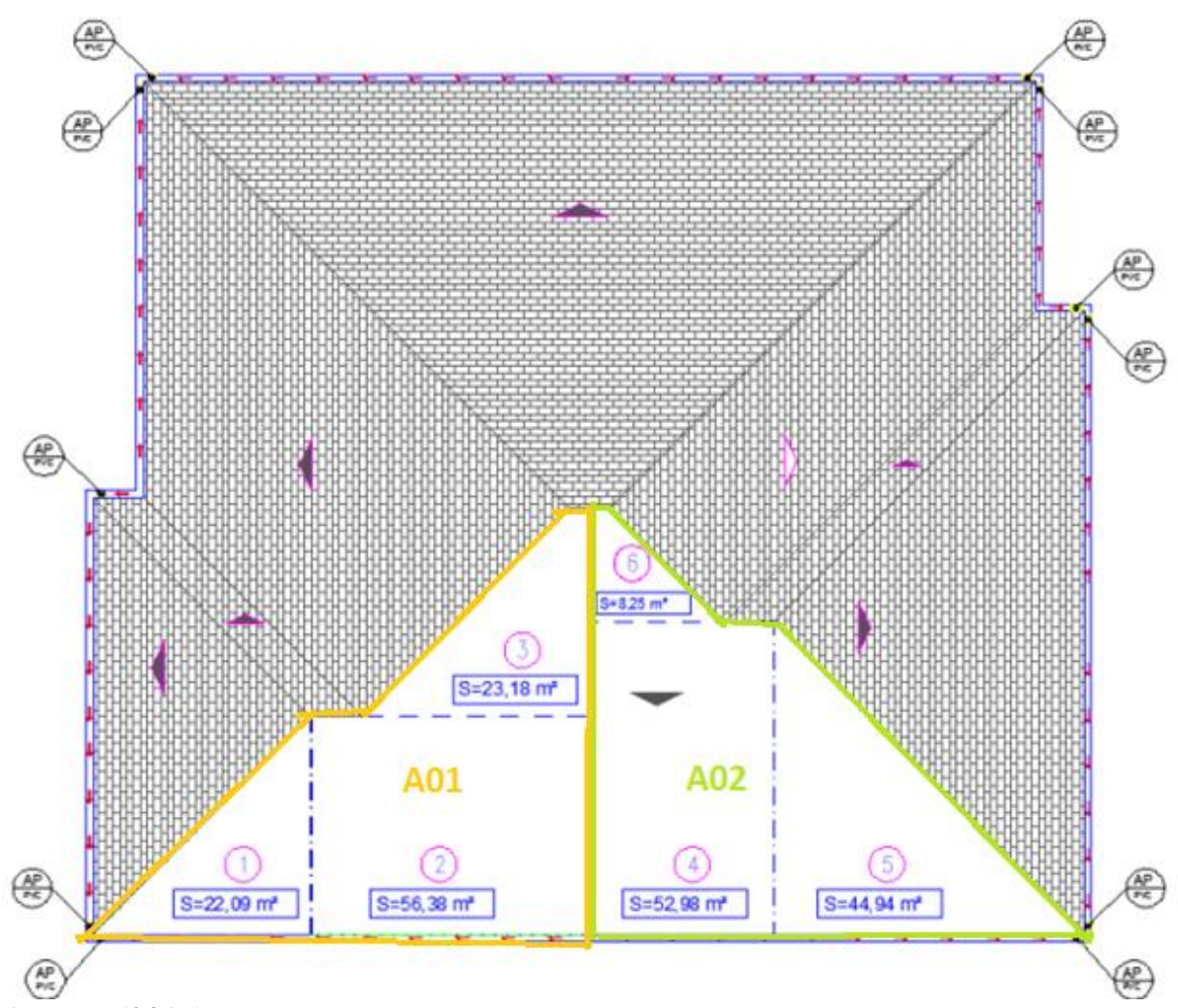

Fonte: Autores (2019).

\subsubsection{DIMENSIONAMENTO DO CONDUTOR VERTICAL E CALHA}

Considerando período de retorno de 5 anos e intensidade pluviométrica de $125 \mathrm{~mm} / \mathrm{h}$, o resultado da vazão de projeto é $221,19 \mathrm{~L} / \mathrm{min}$. Com área de contribuição de $106,17 \mathrm{~m}^{2}$, o diâmetro do condutor vertical corresponde a $75 \mathrm{~mm}$.

Com período de retorno de 25 anos e com intensidade pluviométrica de $180 \mathrm{~mm} / \mathrm{h}$, a vazão de projeto corresponde a 318,51 L/min, sendo o diâmetro do condutor vertical de $100 \mathrm{~mm}$.

Adotou-se para ambos os cenários convencionais a calha do projeto original, possuindo dimensões de 160 x $80 \mathrm{~mm}$, suportando uma vazão de 577,42 L/min.

\subsection{DIMENSIONAMENTO SINFÔNICO - GALPÃO INDUSTRIAL}

O suporte técnico da empresa parceira, repassou apenas os resultados encontrados. Para o cálculo do sistema sifônico, independente do cenário, todas as calhas foram consideradas com dimensões de 400 x $150 \mathrm{~mm}$.

Com as informações necessárias e, a partir da equação do método racional, determinou-se as vazões de projeto para cada captador EPAMS DN100.

\subsubsection{DIMENSIONAMENTO DO SISTEMA SIFÔNICO COM DIFERENTES PERÍODOS DE RETORNO E LÂMINA D'ÁGUA}

As Figura 03 a 06 ilustram o posicionamento dos captadores na cobertura e as informações referentes a cada cenário. 
FIGURA 03 - Captadores para lâmina de 55 mm e 5 anos

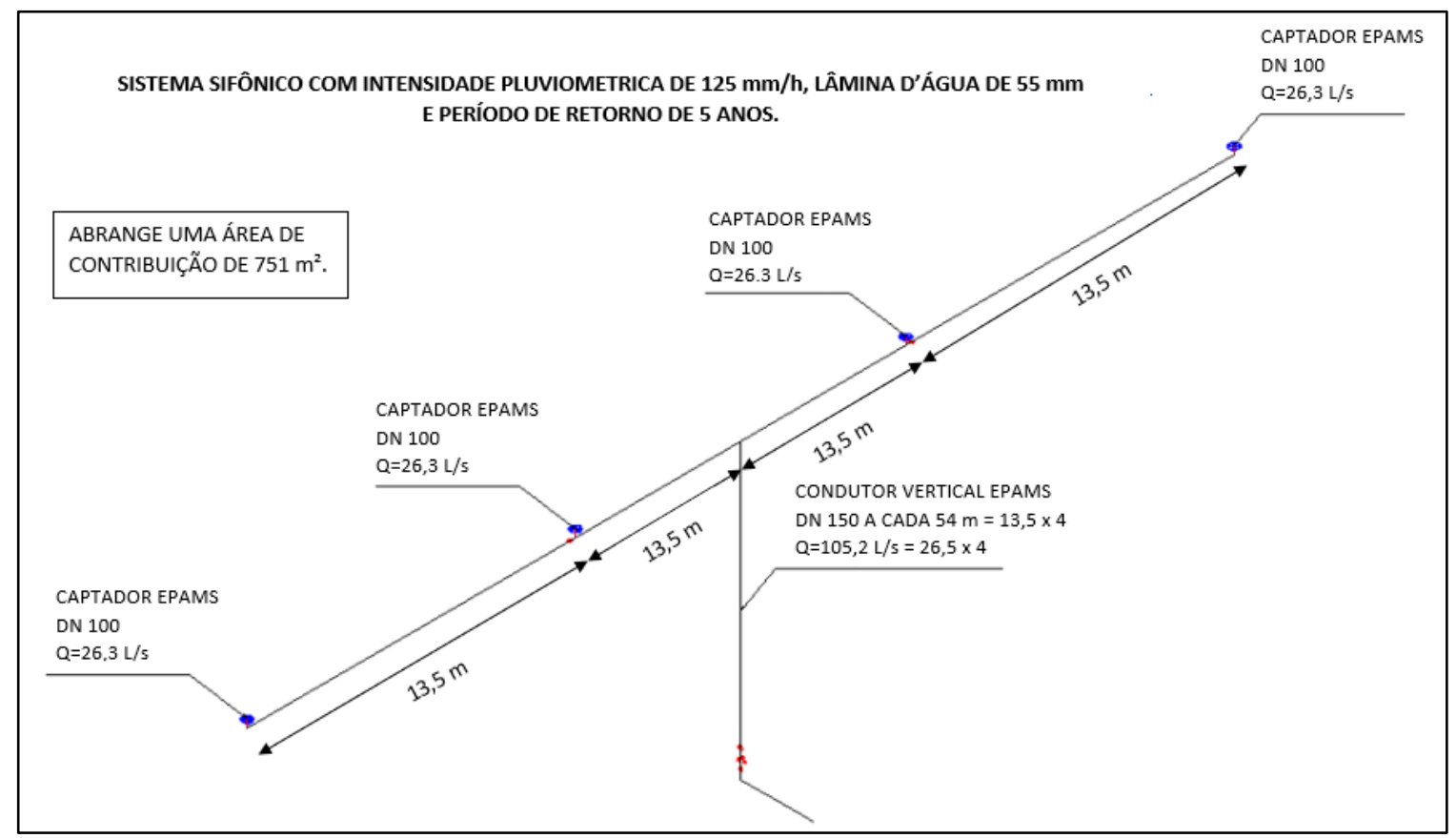

Fonte: Autores (2019).

FIGURA 04 - Captadores para lâmina de 100mm e 5 anos

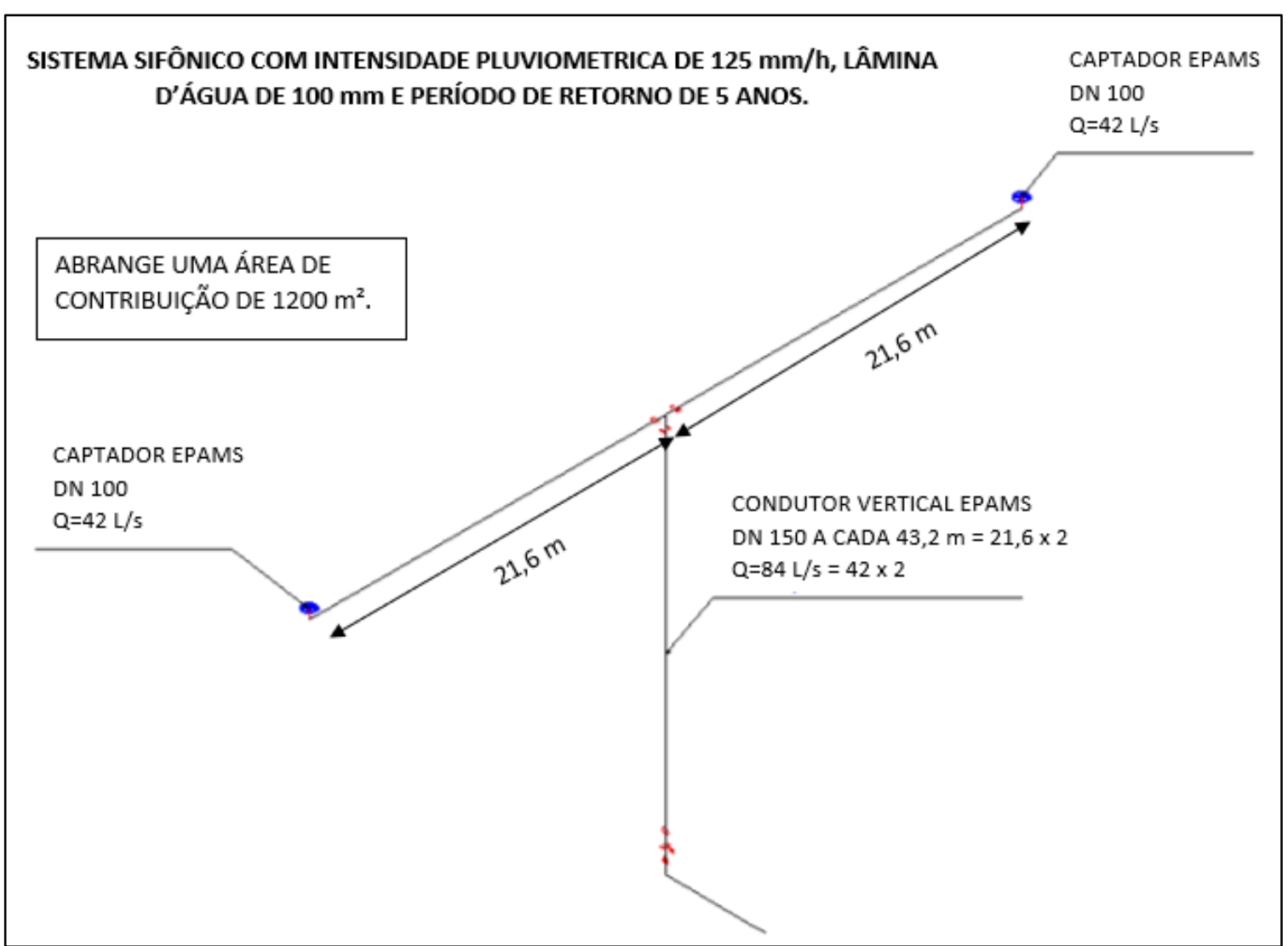

Fonte: Autores (2019). 
FIGURA 05 - Captadores para lâmina de 55mm e 25 anos

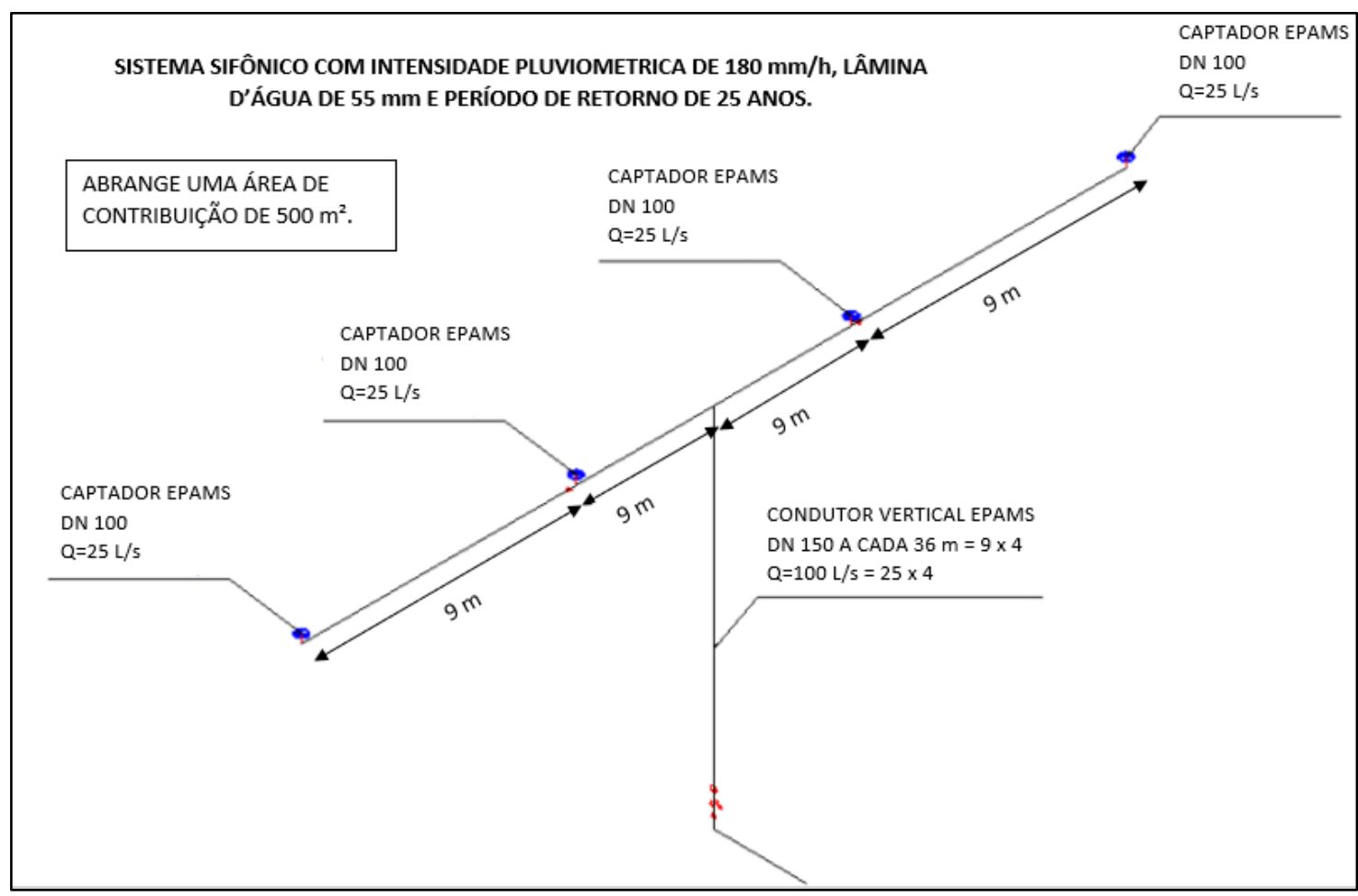

Fonte: Autores (2019).

FIGURA 06 - Captadores para lâmina de 100mm e 25 anos

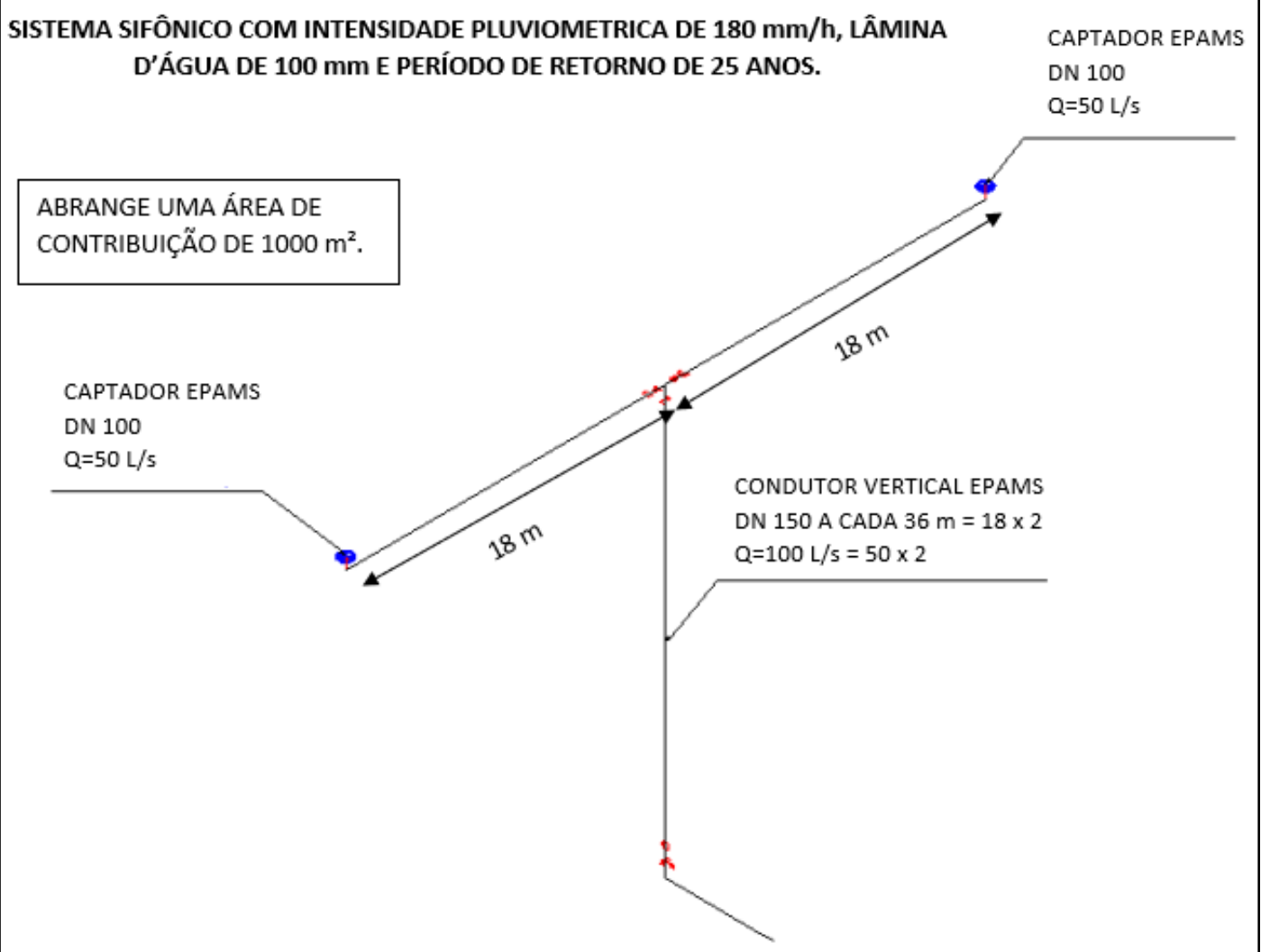

Fonte: Autores (2019). 


\subsection{DIMENSIONAMENTO SIFÔNICO - COBERTURA HOSPITALAR}

A empresa parceira repassou estudos do dimensionamento com período de retorno de 25 anos e lâmina d'água de $55 \mathrm{~mm}$, única situação atendente da vazão mínima para funcionamento do sistema. As calhas possuem dimensões de 400 x $150 \mathrm{~mm}$. Nas demais possibilidades os condutores não funcionaram adequadamente devido as pequenas dimensões da cobertura, impossibilitando obter altura mínima da lâmina d'água dentro da calha, necessitando vazão mínima de $2 \mathrm{~L} / \mathrm{s}$ para inicial o escoamento sifônico.

Na Figura 07 estão informadas a área de influência (S) de cada cobertura, além do diâmetro e vazão recolhida de cada captador.

FIGURA 07 - Captadores para a cobertura hospitalar

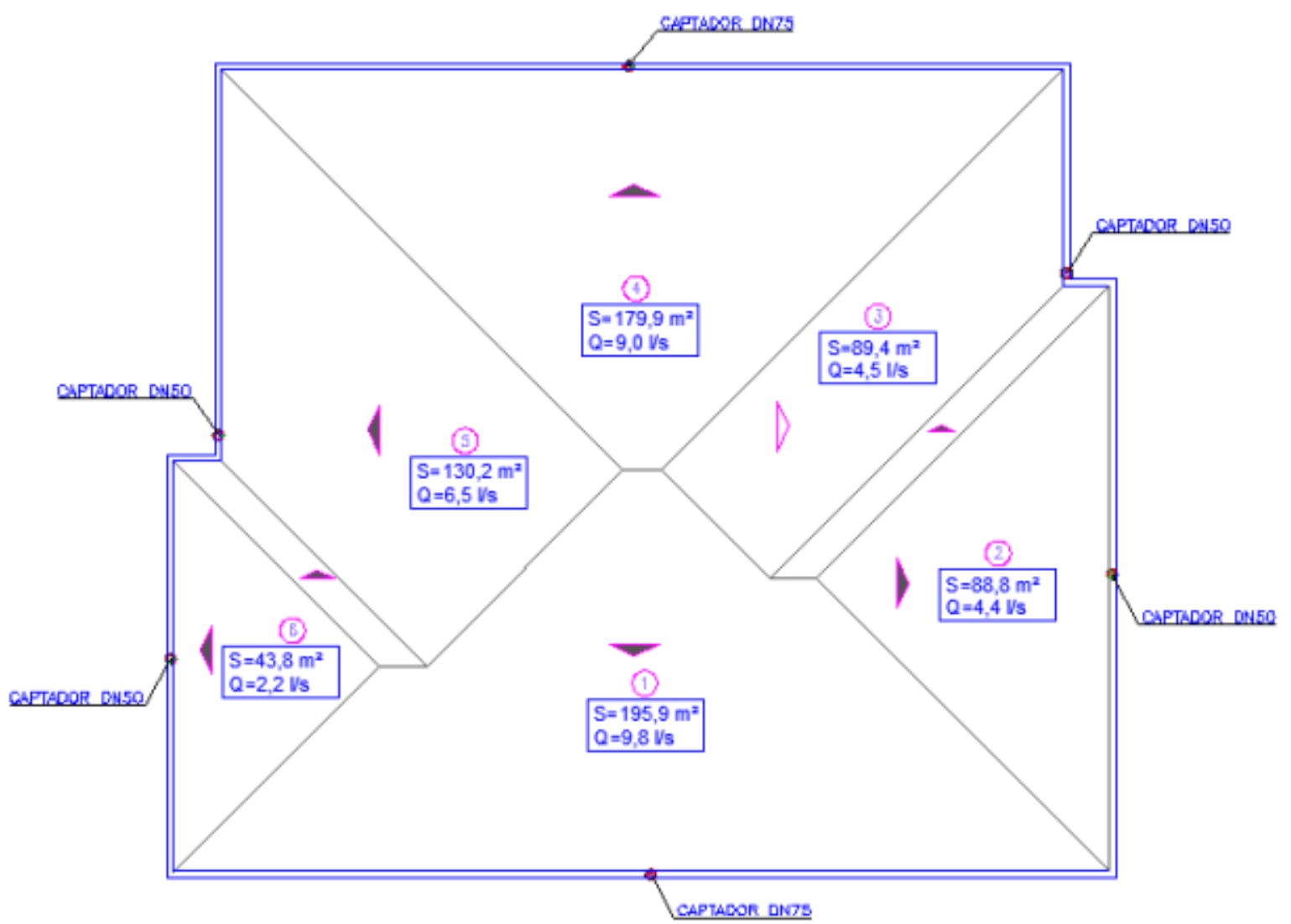

Fonte: Autores (2019).

\subsection{ANÁLISE ECONÔMICA}

\subsubsection{SISTEMA CONVENCIONAL E SISTEMA SIFÔNICO}

Com todos os dimensionamentos, peças, materiais e custos individuais elaborou-se um comparativo de custos para o sistema convencional e sifônico, respectivamente apresentados nos Quadro 03 e 04. 
QUADRO 03 - Custos dos conjuntos dos sistemas convencionais

\begin{tabular}{|c|c|c|c|c|}
\hline \multirow{2}{*}{ Parâmetros } & \multicolumn{2}{|c|}{ GALPÃO INDUSTRIAL } & \multicolumn{2}{c|}{ COBERTURA HOSPITALAR } \\
\cline { 2 - 5 } & $\mathbf{1 2 5} \mathbf{~ m m} / \mathbf{h}$ & $\mathbf{1 8 0} \mathbf{~ m m} / \mathbf{h}$ & $\mathbf{1 2 5} \mathbf{~ m m} / \mathbf{h}$ & $\mathbf{1 8 0} \mathbf{~ m m} / \mathbf{h}$ \\
\hline Calhas & $\mathrm{R} \$ 9.293,34$ & $\mathrm{R} \$ 10.377,57$ & $\mathrm{R} \$ 1.829,06$ & $\mathrm{R} \$ 1.829,06$ \\
\hline Condutores verticais & $\mathrm{R} \$ 19.215,84$ & $\mathrm{R} \$ 27.435,72$ & $\mathrm{R} \$ 1.670,76$ & $\mathrm{R} \$ 2.808,00$ \\
\hline TOTAL & $\mathrm{R} \$ 28.509,18$ & $\mathrm{R} \$ 37.813,29$ & $\mathrm{R} \$ 3.499,82$ & $\mathrm{R} \$ 4.637,06$ \\
\hline
\end{tabular}

Fonte: Autores (2019).

QUADRO 04 - Custos dos conjuntos dos sistemas sinfônicos

\begin{tabular}{|c|c|c|c|c|c|}
\hline \multirow{3}{*}{$\begin{array}{c}\text { Parâmetros } \\
\text { Lâmina (mm) }\end{array}$} & \multicolumn{4}{|c|}{ GALPÃO INDUSTRIAL } & \multirow{3}{*}{\begin{tabular}{|c|}
$\begin{array}{c}\text { COBERTURA } \\
\text { HOSPITALAR }\end{array}$ \\
$\mathbf{1 8 0} \mathbf{~ m m} / \mathbf{h}$ \\
55 \\
\end{tabular}} \\
\hline & \multicolumn{2}{|c|}{$125 \mathrm{~mm} / \mathrm{h}$} & \multicolumn{2}{|c|}{$180 \mathrm{~mm} / \mathrm{h}$} & \\
\hline & 55 & 100 & 55 & 100 & \\
\hline Calhas & $\mathrm{R} \$ 13.165,53$ & $\mathrm{R} \$ 13.165,53$ & $\mathrm{R} \$ 13.165,53$ & $\mathrm{R} \$ 13.165,53$ & $\mathrm{R} \$ 3.379,78$ \\
\hline $\begin{array}{l}\text { Comprimento } \\
\text { das calhas (m) }\end{array}$ & \multicolumn{4}{|c|}{432} & 110,9 \\
\hline $\begin{array}{l}\text { Condutores } \\
\text { verticais }\end{array}$ & $\begin{array}{c}\mathrm{R} \$ \\
232.498,08 \\
\end{array}$ & $\begin{array}{c}\mathrm{R} \$ \\
212.409,80 \\
\end{array}$ & $\begin{array}{c}\mathrm{R} \$ \\
293.938,92 \\
\end{array}$ & $\begin{array}{c}\mathrm{R} \$ \\
205.642,76 \\
\end{array}$ & $\mathrm{R} \$ 25.347,90$ \\
\hline TOTAL & $\begin{array}{c}\mathrm{R} \$ \\
245.663,61 \\
\end{array}$ & $\begin{array}{c}\mathrm{R} \$ \\
225.575,33\end{array}$ & $\begin{array}{c}\mathrm{R} \$ \\
307.104,45\end{array}$ & $\begin{array}{c}\mathrm{R} \$ \\
218.808,29 \\
\end{array}$ & $\mathrm{R} \$ 28.727,68$ \\
\hline
\end{tabular}

Fonte: Autores (2019).

O sistema sifônico, tem maior lâmina d'água dentro da calha, quanto maior a altura, maior a vazão transportada pelo tubo, maior a pressão de sucção gerada, garantindo a eficiência do conjunto. Os valores obtidos na lâmina de $100 \mathrm{~mm}$ acima do captador são menores comparando com $55 \mathrm{~mm}$.

\section{CONCLUSÕES OU CONSIDERAÇÕES FINAIS}

Avaliando dois tipologias de sistemas prediais de águas pluviais (convencional e sifônico) para quatro cenários de estudo, verificou-se que o sistema convencional tem vantagens de baixo custo das peças e drenagem de qualquer vazão, até atingir a capacidade máxima. Por outro lado, o sistema sifônico têm vantagens de flexibilidade de traçado, diminuição das caixas de passagem/caixas de inspeção, possibilita grandes espaçamentos entre condutores verticais, redução dos diâmetros nominais e da declividade podendo esta ser nula, coleta rápida e rapidez na montagem.

Quanto maior a área drenada e a altura dos condutores verticais, maior deve ser a resistência do material, no caso da EPAMS utiliza-se ferro fundido, conectados por juntas próprias. A execução pode ser feita rapidamente, diferente do convencional precisando de juntas coladas ou elásticas.

Também diminui a quantidade de condutores verticais das fachadas com calhas externas, melhorando o visual. Já o convencional possui mais condutores verticais com descidas, normalmente junto aos pilares e grandes diâmetros nominais.

Os captadores do sistema sifônico, possuem vazão mínima para iniciarem o funcionamento de $2,0 \mathrm{~L} / \mathrm{s}$, antes disso funcionam de forma convencional. No caso do estudo realizado, a cobertura hospitalar possui pequena área de coleta, não atingindo a vazão mínima de sifônagem, funcionando, assim como conduto livre. 
Em contrapartida, o sistema sifônico possui bom aproveitamento em coberturas com dimensões e traçados grandes.

Quanto ao custo, o sistema sifônico apresentou maiores valores comparado ao convencional com tubulações de PVC. Para a menor cobertura, a aplicação do sistema resultou em um custo de material 6 vezes mais elevado. No galpão industrial, obteve-se um custo entre 6 a 8 vezes maior que o sistema convencional.

\section{REFERÊNCIAS}

ASSOCIAÇÃO BRASILEIRA DE NORMAS TÉCNICAS. NBR10844: Instalações prediais de águas pluviais. Rio de Janeiro, 1989.

SAINT GOBAIN, EPEMS, sistema anti-vórtice de captação e drenagem de águas pluviais. Disponível em: https://www.sgpam.com.br/artigos/epams-sistema-antivortice-de-captacao-edrenagem-de-aguas-pluviais. Acesso em: 15 jun. 2019

SINAPI. Disponível em: <http://www.caixa.gov.br/poder-publico/apoiopoderpublico/sinapi/Paginas/default.aspx>. Acesso em: 16 jun 2019.

ULMETA. Tetometal Construções Ltda. Disponível em: http://www.sulmeta.com.br/. Acesso em: 15 jun. 2019

VALSIR. Grupo empresarial Valsir, Disponível em: http://www.valsir.it/en/panoramicaraiplus/prodotti/raiplus/panoramica-rainplus. Acesso em: 17 jun.2019 